\title{
Positive Model Theory and Amalgamations
}

\author{
Mohammed Belkasmi
}

\begin{abstract}
We continue the analysis of foundations of positive model theory as introduced by Ben Yaacov and Poizat. The objects of this analysis are $h$-inductive theories and their models, especially the "positively" existentially closed ones. We analyze topological properties of spaces of types, introduce forms of quantifier elimination, and characterize minimal completions of arbitrary $h$-inductive theories. The main technical tools consist of various forms of amalgamations in special classes of structures.
\end{abstract}

\section{Introduction}

Positive model theory is the study of $h$-inductive theories through their models, especially those that are existentially closed, and their type spaces using positive logic. It was initiated by Ben Yaacov in [1], [2] following the line of research on universal theories carried out by Shelah [10], Hrushovski [5], and Pillay [7]. In its current form, positive model theory was introduced by Ben Yaacov and Poizat in [3]. In [8] and [9], Poizat analyzed the topology of type spaces and introduced the notion of positive elementary extension.

In this article, our ultimate goal is to refine the analysis of classes of structures following the line of research of Ben Yaacov and Poizat. The principal subjects will be universal extensions, topological properties of type spaces, quantifier elimination, and connections of these with classes of structures. A recurrent theme will be amalgamation in various classes of structures. Frequently, these structures will be model companions of an $h$-inductive theory or nonelementary classes. The amalgamation analysis consists frequently in verifying that certain classes of models form amalgamation bases (Definition 7), a notion borrowed from Hodges [4] and Pillay [7].

The models of an $h$-inductive theory that are amalgamation bases are those that best represent this theory. In our context, the positively existentially closed (pec

Received January 30, 2012; accepted August 12, 2012

2010 Mathematics Subject Classification: Primary 03C95; Secondary 03C48, 03C10, $03 \mathrm{C} 52$

Keywords: positive model theory, $h$-inductive theories, existentially closed models, amalgamation, elimination of quantifiers, topology of type spaces

(C) 2014 by University of Notre Dame 10.1215/00294527-2420648 
for short) models are typical examples of this property. The analysis of quantifier elimination will show that under additional hypotheses, this representational power is shared by larger classes of models of an $h$-inductive theory: the $h$-maximal and the positively existentially closed ones (Section 5). In general, these particular models do not form elementary classes. We will see in a future work that the pec class is an abstract elementary class in the sense of Shelah, which allows a comparison between the two studies.

The article is organized as follows. In Section 2, after revising the foundations of the subject, we will introduce the notion of universal extension that will be used in verifying the existence of "large" models, in particular models whose classes allow amalgamation. Section 3 is devoted entirely to amalgamation in various classes of models. In particular, we will prove a characterization in terms of universal extensions (Theorem 1). Section 4 contains a first application of the amalgamation techniques developed in the third section, especially of those in Section 3.2: we will analyze the preservation of topological properties of type spaces in substructures and elementary extensions. In Section 5, we will analyze various aspects of quantifier elimination. In particular, we will use the notion of a positive Robinson theory, an $h$-inductive theory that allows a certain kind of quantifier elimination. In Section 6, we will finish by studying completions of $h$-inductive theories. This will set the foundations for the first steps of a work in preparation on positive stability and simplicity.

\section{Positive Model Theory}

2.1 Basics Positive logic is a branch of first-order mathematical logic whose specific property is not using negation. This restricts the available set of first-order formulas to the set of the positive ones obtained from atomic formulas using $\vee, \wedge, \exists$ as logical operators and quantifier, respectively. Eventually, a positive first-order formula is of the form $\exists \bar{y} f(\bar{x}, \bar{y})$, where $f(\bar{x}, \bar{y})$ is quantifier-free. The special symbol $\perp$ denoting the antilogy needs to be added. The rest of this section is devoted to recalling various definitions and notions of positive logic. For further details, [3] is a sufficiently complete reference.

As in the first-order logic with negation, a sentence is a formula without free variables. A sentence is said to be h-universal if it is the negation of a positive sentence, that is, if it is of the form $\neg \exists \bar{x} f(\bar{x})$, or equivalently, $\forall \bar{x} \neg f(\bar{x})$, where $f(\bar{x})$ is quantifier-free and positive. The conjunction of two $h$-universal sentences is equivalent to an $h$-universal sentence. The same is true for their disjunction.

A sentence is said to be simple h-inductive if it can be written in the form

$$
\forall \bar{x}[\exists \bar{y} f(\bar{x}, \bar{y}) \leftarrow \exists z g(\bar{x}, \bar{z})],
$$

where $f$ and $g$ are quantifier-free and positive. In prenex normal form, such a sentence is of the form

$$
\forall \bar{u} \exists \bar{v}(\neg \varphi(\bar{u}) \vee \psi(\bar{u}, \bar{v})),
$$

where $\varphi$ and $\psi$ are quantifier-free and positive. It follows that the disjunction of two simple $h$-inductive sentences is still simple $h$-inductive. An $h$-inductive sentence is a finite conjunction of simple $h$-inductive sentences. The conjunction and disjunction of two $h$-inductive sentences is still $h$-inductive.

A first-order theory is said to be $h$-inductive if it is formed by $h$-inductive sentences. In the particular case when they are all $h$-universal, such a theory is called 
$h$-universal. The $h$-inductive theories are the objects of analysis of positive model theory.

Let $L$ be a first-order language, and let $M$ and $N$ be two $L$-structures. A mapping from $M$ to $N$ is a homomorphism if, for every tuple $\bar{m}$ extracted from $M$ ( $\bar{m} \in M$ for short) and for every atomic formula $\varphi, M \models \varphi(\bar{m})$ implies $N \models \varphi(h(\bar{m}))$. In such a case, $N$ is said to be a continuation of $M$. A homomorphism is an embedding whenever, for every atomic formula, $\varphi M \models \varphi(\bar{m})$ if and only if $N \models \varphi(h(\bar{m}))$; it is an immersion whenever $\bar{m}$ and $h(\bar{m})$ satisfy the same positive formulas.

A positive compactness theorem was proved by Ben Yaacov and Poizat, and we will refer to its following form as "positive compactness."

Fact 1 ([3, Corollaire 4]) An $h$-inductive theory is consistent if and only if every finite subset of it is consistent.

A class of structures is said to be inductive if it is closed with respect to inductive limits of homomorphisms. It is easy to verify that the class of models of an $h$-inductive theory is inductive. Théorème 23 of [3] shows that this is indeed a characterization.

Fact 2 ([3, Théorème 23]) The class of models of a first-order theory is inductive if and only if it is axiomatized by $h$-inductive sentences.

2.2 Positively existentially closed models The notion of pec models is fundamental in positive model theory.

Definition 1 Let $L$ be a first-order language. A member $M$ of a class $C$ of $L$ structures is said to be positively existentially closed in $C$ if every homomorphism from $M$ into an element of $C$ is an immersion.

The following fact will be used without mention together with Fact 2 to verify that every model of an $h$-inductive theory has a pec continuation.

Fact 3 ([3, Théorème 1]) Every member of an inductive class of models has an existentially closed continuation in the same class.

Definition 2 ([3]) Two $h$-inductive theories are said to be companions if they have the same $h$-universal consequences.

Companionship of models is characterized using the notion of a pec model.

Fact 4 ([3, Lemme 7]) Two $h$-inductive theories are companions if and only if they have the same pec models.

The analysis of $h$-inductive theories in [3] as well as Fact 2 above show that an $h$ inductive theory $T$ has a maximal companion, denoted $T k$ and called the Kaiser envelope of $T$; it is the $h$-inductive theory of the pec models of $T$-equivalently, $T k$ is the set of all $h$-inductive sentences true in the pec models of $T$. At the opposite extreme, $T$ has a minimal companion, denoted $T u$, formed by its $h$-universal consequences. When parameters from a certain set $A$ are allowed, the notation will be $T u(A)$ and $T k(A)$.

An $h$-inductive theory $T$ is said to be model-complete if all its models are pec, in other words, if the class of pec models is axiomatized by the Kaiser envelope $T k$. An example of a model-complete theory is that of algebraically closed fields of a fixed characteristic in the language of fields. 
Fact 5 ([3, Lemme 5]) Let $T$ be an $h$-inductive theory, and let $T u$ be its $h$ universal consequences. Then a structure has a continuation that is a model of $T$ if and only if it is a model of $T u$.

It follows from this fact that every structure that has a pec continuation that is a model of $T$ is a model of $T u$.

Examples 1 Let $L=\{R\}$, where $R$ is a relation symbol and $T$ the $h$-inductive theory that states that $R$ is an equivalence relation. Then $T$ has a unique pec model which is the model with a unique equivalence class consisting of a single element.

Let $L$ be the language with two relation symbols $P$ and $Q$, and let $T$ be the $h$-universal theory $\{\neg \exists x, y P(x) \wedge Q(y)\}$. Then $T$ has exactly two pec models: $\{A\}=\{a\}$ such that $A \models P(a)$ and $B=\{b\}$ such that $B \models Q(b)$.

Let $T$ be the $h$-inductive theory of algebraically closed fields of characteristic $p$ in the language $L=\left\{+,-, \cdot,{ }^{-1}, 0,1\right\}$. A model $A$ of $T$ is pec if and only if it is algebraically closed. Thus, the Kaiser envelope of $T$ is the theory of algebraically closed fields of the same characteristic.

Since, in general, every structure that has a continuation which is a model of $T$ is a model of $T u$ (Fact 5), every ring that has a continuation which is a pec model of the theory of fields of characteristic $p$ is a model of the $h$-universal theory of fields of characteristic $p$. To illustrate, the ring of integers has this property. Since two theories of fields of distinct characteristics have distinct pec models, the respective $h$-universal theories determine the characteristics of the fields.

The following conclusion will be useful in various constructions that make use of inductive limits.

Fact 6 ([3, Lemme 12]) The class of pec models of an $h$-inductive theory $T$ is inductive.

A recent result on pec structures has been proved by Almaz Kungozhin.

Fact 7 ([6]) Let $L$ be a relational language, and let $T$ be a finitely axiomatizable $h$-universal theory. Then the class of pec models of $T$ is elementary.

2.3 Type spaces As in every model-theoretic analysis, the notion of type is fundamental in positive model theory. The positive context forces the types under analysis to consist of positive formulas and requires the following subtler definition.

Definition 3 ([3], [9, Section 3]) Let $T$ be an $h$-inductive theory in a language $L$. An $n$-type is a maximal set of positive formulas in $n$ variables that is consistent with $T$ or with one of its companions.

An $n$-type with parameters in $M$ is a maximal set of positive formulas in $n$ variables with parameters in $M$, that is consistent with $T(M)$, equivalently with $T k(M)$.

It is worth emphasizing that one can also define a positive type as the set of positive formulas satisfied by an element of a pec model of an $h$-inductive theory. This allows us to characterize the pec models by the maximality of the sets of positive formulas that tuples of their elements satisfy.

Fact 8 ([3, Lemme 13]) A model $A$ of $T$ is pec if and only if, for every $\bar{a} \in A$, the set of positive formulas satisfied by $\bar{a}$ is a type. 
From this fact, one deduces that if $A$ is pec and $\bar{a} \in A$ such that $A \models \neg \varphi(\bar{a})$, where $\varphi$ is a positive formula, then there exists $\psi$ such that $A \models \psi(\bar{a})$ and $T \vdash \neg \exists x(\varphi(\bar{x}) \wedge \psi(\bar{x}))$.

Following the preceding line of thought, when $A$ is a model of an $h$-inductive theory $T$ and $\bar{a} \in A$, we note that $F_{A}(\bar{a})$ is the set of formulas satisfied by $\bar{a}$ in $A$. Thus, if $A$ is not a pec model, $F_{A}(\bar{a})$ is not necessarily a type (a maximal set).

The usual notation is adopted to denote types. We denote by $S_{n}(T)$ (resp., $S_{n}(M)$ ) the space of $n$-types of a theory $T$ (resp., of the theory $T(M)$ with parameters in $M)$. An $n$-type of $S_{n}(T)$ (resp., of $S_{n}(M)$ ) has a realization in a pec model of $T$ (resp., in an elementary extension of $M$ ).

One defines on $S_{n}(A)$ a topology of which the basis of closed sets is the set of $F_{f}$, where $f$ ranges over the entire set of positive formulas, and

$$
F_{f}=\left\{p \in S_{n}(A) \mid p \vdash f\right\} .
$$

The space of positive types is compact (quasicompact in some mathematical cultures) by Fact 1, but it is not necessarily Hausdorff. In [9], Poizat analyzed consequences of the lack of the Hausdorff property. In Section 4, we will concentrate on this problem in a systematic way.

2.4 Positive elementary extensions The notion of elementary extension in positive model theory was introduced and analyzed in [8].

Definition 4 ([8, Section 2]) Let $M$ and $N$ be two $L$-structures such that $N$ is a continuation of $M$. The structure $N$ is an elementary extension of $M$ if $N$ is a pec member of the class of models of the $h$-universal theory $T u(M)$ in the language $L(M)$.

In [8], Poizat proves the following characterization of positive elementary extensions.

Fact 9 ([8, Lemme 1]) A continuation $N$ of $M$ is an elementary extension of the latter if and only if the following two conditions are satisfied:

1. $M$ is immersed in $N$;

2. for every $\bar{b} \in N$, and every positive existential $L$-formula $f(\bar{x})$ not satisfied by $\bar{b}$ in $N$, there exists a positive existential formula $g(\bar{x}, \bar{a})$, with parameters $\bar{a} \in M$, that is satisfied by $\bar{b}$ and contradictory with $f(\bar{x})$ : the sentence $\neg(\exists \bar{x}, \bar{y}, \bar{z})(f(\bar{x}, \bar{y}) \wedge g(\bar{x}, \bar{z}, \bar{a}))$ belongs to $T u(M)$, where $f(\bar{x}, \bar{y})$ and $g(\bar{x}, \bar{z}, \bar{a})$ are quantifier-free.

2.5 Universal extensions The notion of universal extension is reminiscent of universal objects in category theory. In our context, inductive limits of universal extensions generalize the notion of saturation and are also relevant for relationships with abstract elementary classes (see Section 6).

In this section, we will analyze properties of this notion, and in the next one, we will obtain a characterization of structures that admit a universal extension. In an article in preparation, we will use this notion to obtain "monster models" in connection with positive stability and simplicity.

Definition 5 Let $A$ and $B$ be models of an $h$-inductive theory $T$, and let $h$ be a homomorphism from $A$ to $B$. The pair $(B, h)$ is said to be a universal extension of $A$ if, for every model $C$ of $T$ of cardinality at most $|A|$ such that there is a homomorphism from $A$ to $C$, there exists a homomorphism $g$ from $C$ to $B$ such that the 
following diagram commutes:

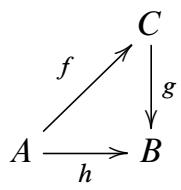

Remark 1 Let $(B, h)$ be a universal extension of $A$, and let $g$ be a homomorphism from $B$ to a model $C$ of $T$. Then $(C, g \circ h)$ is also a universal extension of $A$. In particular, $A$ admits a universal extension $\left(B_{e}, h^{\prime}\right)$, where $B_{e}$ is a pec model of $T$.

The following lemma is a positive form of the descending Löwenheim-Skolem theorem. It is a slightly modified version of [3, Lemma 11].

Lemma 1 Let $T$ be an h-inductive theory, let $A$ be a model of $T$, and let $B$ be a subset of $A$. Then there exists a model $B^{\star}$ of $T$ of cardinality at most $\max (|B|,|L|)$ that contains $B$ and that is immersed in $A$.

Proof The proof is the same as that of [3, Lemma 11]. It suffices to note that the structure $B^{\star}$ obtained at the end of the construction in [3] is a model of $T$. In this vein, suppose that

$$
T \vdash \forall \bar{x}[\exists \bar{y} \varphi(\bar{x}, \bar{y}) \rightarrow \exists \bar{z} \psi(\bar{x}, \bar{z})] .
$$

If $B^{\star} \models \exists \bar{y} \varphi(\bar{a}, \bar{y})$, where $\bar{a} \in B^{\star}$, then $A \models \exists \bar{y} \varphi(\bar{a}, \bar{y})$ and $A \models \exists \bar{z} \psi(\bar{a}, \bar{z})$. One then deduces from the construction of $B^{\star}$ that $B^{\star} \models \exists \bar{z} \psi(\bar{a}, \bar{z})$. Hence, $B^{\star} \models T$.

Definition 6 Let $T$ be an $h$-inductive theory, and let $\alpha$ be an ordinal. A universal chain of length $\alpha$ of $T$ is an inductive family of models $\left\{A_{i}: i<\alpha\right\}$ (resp., $\left\{A_{i}: i \leq \alpha\right\}$ if $\alpha$ is a successor ordinal) of $T$ with a family of homomorphisms $\left\{f_{i j}: i \leq j<\alpha\right\}$ (resp., $\left\{f_{i j}: i \leq j<\alpha\right\}$ if $\alpha$ is a successor ordinal) such that for every ordinal $\beta<\alpha,\left(A_{\beta+1}, f_{\beta, \beta+1}\right)$ is a universal extension of $A_{\beta}$, and that if $\beta \leq \alpha$ is a limit ordinal, then $A_{\beta}$ is the inductive limit of the $A_{i}$ with $i<\beta, f_{i \beta}$ being defined as the canonical mapping from $A_{i}$ into $A_{\beta}$.

Lemma 2 Let $\left\{A_{i}, f_{i j}: i \leq j<\alpha\right\}$ be a universal chain of an $h$-inductive theory $T$. Then for every limit ordinal $i \leq \alpha, A_{i}$ is a pec model of $T$. In this case, if $j \leq i$, then $f_{j i}$ is the canonical mapping $A_{j}$ to $A_{i}$ for inductive limits, and $\left(A_{i}, f_{j i}\right)$ is a universal extension of $A_{j}$.

Proof Let $A_{i}$ be a member of the universal chain with $i$ a limit ordinal. We will first show that $A_{i}$ is a pec model of $T$. As $A_{i}$ is an inductive limit of models of $T$, $A_{i}$ is itself a model of $T$ (see Fact 2). Let now $f$ be a homomorphism from $A_{i}$ to a model $B$ of $T$. Let us suppose that $B \models \varphi(f(\bar{a}))$, where $\varphi$ is a positive formula and $\bar{a} \in A_{i}$. Then there exists $\beta<i$ such that $\bar{a} \in A_{\beta}$ and $\bar{a}=f_{\beta, i}(\bar{a})$.

By Lemma 1, there exists $B^{\prime}$, a model of $T$ generated by $f \circ f_{\beta, i}\left(A_{\beta}\right)$ of cardinality at most $\left|A_{\beta}\right|$ such that $B^{\prime} \models \varphi\left(f \circ f_{\beta, i}(\bar{a})\right)$. As $\left(A_{\beta+1}, f_{\beta, \beta+1}\right)$ is a universal extension of $A_{\beta}$, there exists an homomorphism $h$ from $B^{\prime}$ into $A_{\beta+1}$ such that the 
following diagram commutes:

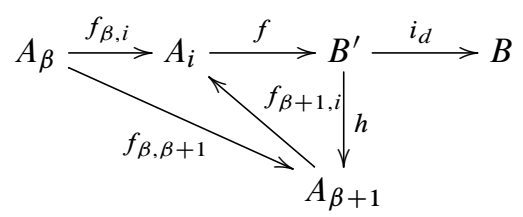

As $B^{\prime} \models \varphi\left(f\left(f_{\beta, i}(\bar{a})\right)\right)$ and $h \circ f \circ f_{\beta, i}=f_{\beta, \beta+1}$, we conclude that $A_{\beta+1} \models$ $\varphi\left(f_{\beta, \beta+1}(\bar{a})\right)$. By the definition of an inductive limit, $f_{\beta+1, i} \circ f_{\beta, \beta+1}=f_{\beta, i}$, and so $A_{i} \models \varphi(\bar{a})$, which implies that $f$ is an immersion. Hence, $A_{i}$ is a pec model of $T$.

We will now show that for every $\beta<i,\left(A_{i}, f_{\beta, i}\right)$ is a universal extension of $A_{\beta}$. Let $C$ be a model of $T$, let $g$ be a homomorphism from $A_{\beta}$ into $C$, and assume that $|C| \leq\left|A_{\beta}\right|$. As $\left(A_{\beta+1}, f_{\beta, \beta+1}\right)$ is a universal extension of $A_{\beta}$, there exist $f$ and $H$ such that the following diagram commutes:

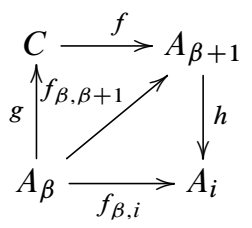

We deduce from the commutativity of the diagram that $f_{\beta, i}=h \circ f_{\beta, \beta+1}=h \circ f \circ g$. The conclusion follows.

\section{Amalgamations}

The possibility of amalgamating the structures in a given class allows a finer study of it. This section continues the analysis of amalgamation techniques initiated in [3]. In Section 3.1, we will introduce and characterize the amalgamation bases following [4] and [7].

The ability to amalgamate, being a property of "maximal" structures, is strongly connected to the analysis of "maximal" $h$-inductive theories. Section 3.2 is devoted to the analysis of amalgamation of models of the Kaiser envelope of an $h$-inductive theory.

\subsection{Amalgamation bases}

Definition $7 \quad$ Let $T$ be an $h$-inductive theory. A model $A$ of $T$ is said to be an amalgamation basis if, for every pair of models $B$ and $C$ of $T$ such that there exist homomorphisms $f$ and $g$ from $A$ to $B$ and $C$, respectively, there exist a model $D$ of $T$ and $f^{\prime}, g^{\prime}$ homomorphisms such that the following diagram commutes:

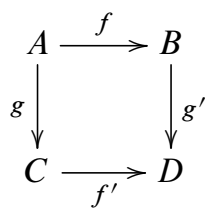

A theory is said to have the amalgamation property if each model of $T$ is an amalgamation basis. 


\section{Examples 2}

- Let $L$ be the relational language $\{P, Q, R\}$, and let $T$ be the $h$-universal theory $\{\neg \exists x(Q(x) \wedge R(x))\}$. Let $A, B, C$ be the three models of $T$ with the following properties: $A=\{a\}, B=\{b\}, C=\{c\}$; we have

$$
A \models P(a), \quad B \models P(b) \wedge Q(b), \quad C \models P(c) \wedge R(c) .
$$

The applications that map $a$ to $b$ and $a$ to $c$ are homomorphisms from $A$ to $B$ and from $A$ to $C$, respectively. But because of the sentence $\neg \exists x(Q(x) \wedge R(x))$, one cannot amalgamate $B$ and $C$. Thus $A$ is not an amalgamation basis.

- A quick recipe to obtain amalgamation bases for an $h$-inductive theory is to use Corollary 1 below. Indeed, the pec models of any $h$-inductive theory are amalgamation bases.

As a reminder, we note that for a structure $A, F_{A}(\bar{a})$ is the set of positive formulas satisfied by $\bar{a}$ in $A$.

Lemma 3 Let $T$ be an h-inductive theory, and let $A$ be a model of $T$. Then the following properties are equivalent:

1. A is an amalgamation basis;

2. for every $\bar{a} \in A$, there exists a unique type in $S(T)$ that contains $F_{A}(\bar{a})$.

Proof $\quad((1) \Rightarrow(2))$ Let $\bar{a} \in A$. We suppose that there exist two distinct types $p \neq q$ in $S(T)$ that contain $F_{A}(\bar{a})$.

We first show that since $p \vdash F_{A}(\bar{a})$, there exist $B$ a pec model of $T$ and $f$ a homomorphism from $A$ to $B$ that maps $\bar{a}$ to $\bar{b}$ that realizes $p$. In this vein, it suffices to show that the family $\Gamma=T \cup \operatorname{Diag}^{+}(A) \cup p(\bar{a})$ is consistent. Let $A^{\prime}$ be a model of $T$ that realizes $p$ with $\bar{a}^{\prime}$ and $\varphi(\bar{a}, \bar{m}) \in \operatorname{Diag}^{+}(A)$. Then $\exists \bar{y} \varphi(\bar{x}, \bar{y}) \in F_{A}(\bar{a})$, so $p \vdash \exists \bar{y} \varphi(\bar{x}, \bar{y})$. Hence there exists $\bar{c}^{\prime}$ in $A^{\prime}$ such that $A^{\prime} \models \varphi\left(\bar{a}^{\prime}, \bar{c}^{\prime}\right)$, from which follows that the family $\Gamma$ is consistent. Let $B^{\prime}$ be a model of $\Gamma$, and let $B$ be a pec model of $T$ that is a continuation of $B^{\prime}$. Then there is a homomorphism from $A$ into $B$ and $\bar{b}$, which is the image of $\bar{a}$ in $B$ that realizes $p$.

Similarly, there exist $C$ a pec model of $T$ and a homomorphism $g$ from $A$ to $C$ which maps $\bar{a}$ to $\bar{c}$, a realization of $q$. Since $A$ has the amalgamation property, there exists $D$ a model of $T$ such that the following diagram commutes:

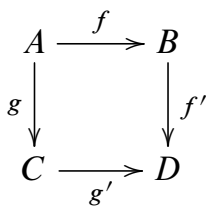

Thus $f^{\prime}(\bar{b})=g^{\prime}(\bar{c})$, and hence $p=q$, a contradiction.

((ii) $\Rightarrow$ (i)) Let

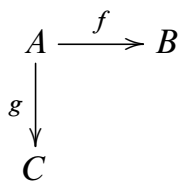

with $A$ a model of $T$. Let $B, C$ be pec models of $T$, and let $f, g$ be homomorphisms as in the diagram. Suppose that one cannot amalgamate $f$ and $g$. This would mean 
that the following $h$-inductive theory is inconsistent:

$$
T \cup \operatorname{Diag}^{+}(A) \cup \bigcup_{\bar{a} \in A} F_{B}(f(\bar{a})) \cup \bigcup_{\bar{a} \in A} F_{C}(g(\bar{a})) .
$$

By positive compactness, there exists a finite fragment of this theory that is inconsistent. It follows that there exists $\bar{a} \in A$ such that $F_{B}(f(\bar{a}))$ and $F_{C}(g(\bar{a}))$ (which are types as $B$ and $C$ are pec models of $T$ ) are contradictory. This contradicts hypothesis (ii).

Theorem 1 Let A be a model of an h-inductive theory T. Then A has a universal extension if and only if $A$ is an amalgamation basis.

Proof Let us suppose that $A$ has a universal extension $(B, h)$ and show that $A$ is an amalgamation basis. Let $A_{i} \models T, i=1,2$ be two continuations of $A$ by the homomorphisms $f_{i}$. In order to verify the amalgamation property, it suffices to show that the following family is consistent:

$$
\Gamma=T \cup \operatorname{Diag}^{+}\left(A_{1}\right) \cup \operatorname{Diag}^{+}\left(A_{2}\right)
$$

by interpreting the parameters from $A$ by the same symbols in $A_{1}$ and $A_{2}$.

We fix a subset $T \cup \Gamma_{1} \cup \Gamma_{2}$, where the $\Gamma_{i}$ are finite subsets of $\operatorname{Diag}^{+}\left(A_{i}\right)$ for $i=1,2$. Let $\bar{a}_{i}$ be the parameters from $A_{i}$ that are used in $\Gamma_{i}$. By Lemma 1 , there exists a model $B_{i}$ of $T \cup \Gamma_{i}$ that contains $A \cup\left\{\bar{a}_{i}\right\}$ and that has the same cardinality as $A$. We will denote by $g_{i}$ the homomorphism from $A$ into $B_{i}$ defined by $g_{i}(a)=f_{i}(a)$ for every $a \in A$. Then by definition of universal extensions, one has the following diagram:

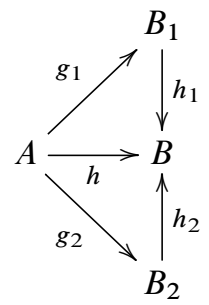

This implies that $B$ is a model of the set $T \cup \Gamma_{1} \cup \Gamma_{2}$. By positive compactness, $\Gamma$ is consistent. This proves the existence of the amalgamation being sought.

In order to prove the other implication, we assume that $A$ is an amalgamation basis. We will show that it has a universal extension. Let $\Delta$ be the family of all pairs $(M, f)$ with $M$ a model of $T$ of cardinality at most $|A|$ such that there exists a homomorphism $f$ from $A$ to $M$. By the axiom of choice, we may suppose that $\Delta$ is well ordered. Its order type will be denoted by $\alpha$.

We will construct an inductive family $\left\{A_{\beta}: \beta \leq \alpha\right\}$ of models of $T$ with a coherent family of homomorphisms $\left\{h_{i, j}: i \leq j \leq \alpha\right\}$. The homomorphisms will be indexed by pairs of ordinals up to $\alpha$. The last member of the sequence, $A_{\alpha}$, will be the universal extension that is being sought.

To start the construction, we set $A_{0}=A$, and $h_{0,0}$ is defined as the identity mapping. Since $A$ is an amalgamation basis, there exist $A_{1}$, a model of $T$, and two homomorphisms $h_{0,1}$ and $g_{0}$ from $A_{0}$ to $A_{1}$ and from $M_{0}$ to $A_{1}$, respectively, such 
that the following diagram commutes:

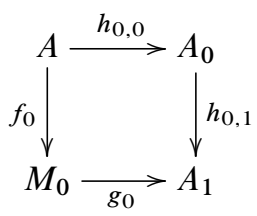

For the inductive step, we assume that the family $\left\{A_{\beta}: \beta<\gamma \leq \alpha\right\}$ with the corresponding homomorphisms has been constructed. If $\gamma$ is a successor of the form $\beta+1$, there exist a model $A_{\gamma}$ of $T$ and homomorphisms $h_{\beta, \beta+1}$ and $g_{\beta}$ such that the following diagram commutes:

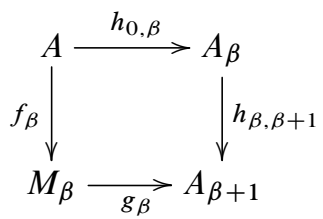

For every $i \leq \beta$, we set $h_{i, \beta+1}=h_{\beta, \beta+1} \circ h_{i, \beta}$. The coherence of the homomorphisms already constructed inductively implies that the new family is still coherent. In other words, we continue to have an inductive family of models of $T$.

If $\gamma$ is a limit ordinal, then one defines $A_{\gamma}$ as the inductive limit of the already constructed inductive family. As for the new homomorphisms, for every $i<\gamma, h_{i, \gamma}$ is the natural mapping from $A_{i}$ to $A_{\gamma}$. The new family of models and homomorphisms is also inductive.

The construction ends when $\alpha$ is reached. By construction, either $\alpha$ is a successor and thus $A_{\alpha}$ is constructed as in the inductive step for successors, or $\alpha$ is a limit ordinal and $A_{\alpha}$ is the inductive limit of the family $\left\{A_{i}: i<\alpha\right\}$.

To finish the proof, we show that $\left(A_{\alpha}, h_{0, \alpha}\right)$ is a universal extension of $A$. Let $M$ be a model of $T$ of cardinality at most $|A|$ such that there exists a homomorphism $f$ from $A$ into $M$. By the definition of the family $\Delta$, there exists $\beta \leq \alpha$ such that $(M, f)=\left(M_{\beta}, f_{\beta}\right)$. If $\beta=\alpha$, then the identity mapping on $A_{\alpha}$ suffices. Otherwise, $\beta<\alpha$ and by construction, the following diagram is commutative:

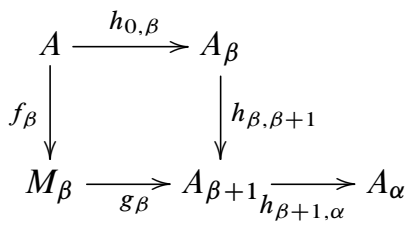

The equalities $h_{0, \alpha}=h_{\beta+1, \alpha} \circ h_{\beta, \beta+1} \circ h_{0, \beta}=h_{\beta+1, \alpha} \circ g_{\beta} \circ f_{\beta}$ that follow from this diagram yield the desired conclusion.

3.2 Amalgamations in models of Kaiser envelopes In earlier works on positive model theory, the existence of amalgamations is frequently analyzed in the context of $h$-universal theories. Here, it will be necessary to extend the context to Kaiser envelopes. To start, we will prove a slightly modified version of the so-called "asymmetric amalgamation" of Ben Yaacov and Poizat in [3, Lemma 8]. 
Lemma 4 Let $A, B, C$ be L-structures, let $g$ be an immersion from $A$ into $B$, and let $h$ be a homomorphism from $A$ to $C$. Then, there exist a model $D$ of $T k(C)$, a homomorphism $g^{\prime}$ from $B$ to $D$, and an immersion $h^{\prime}$ from $C$ into $D$ such that $g^{\prime} \circ g=h^{\prime} \circ h$.

Proof We use the same symbols to name the elements of $A$ in $B$ and $C$. The proof consists in showing that the set

$$
T k(C) \cup \operatorname{Diag}^{+}(B)
$$

of sentences is consistent. In this vein, let $f(\bar{a}, \bar{b})$ be in $\operatorname{Diag}^{+}(B)$ with $\bar{a} \in A$ and $\bar{b} \in B$. Then $A \models \exists \bar{y} f(\bar{a}, \bar{y})$ since $A$ is immersed in $B$. Hence, one can interpret $\bar{b}$ by a tuple $\bar{b}^{\prime} \in A$ such that $A \models f\left(\bar{a}, \bar{b}^{\prime}\right)$. Thus, $C \models f\left(h(\bar{a}), h\left(\bar{b}^{\prime}\right)\right)$. The formula obtained by replacing $h(\bar{a})$ and $h\left(\bar{b}^{\prime}\right)$ by the corresponding constant symbols in the language $L(C)$ belongs to $T k(C)$.

This lemma has the following corollary mentioned in [3] in a different form.

Corollary 1 The pec models of an h-inductive theory are amalgamation bases.

We deduce the following connection with universal extensions.

Corollary 2 Let $A_{e}$ be a pec model of an h-inductive theory. Then $A_{e}$ has a universal extension $\left(B_{e}, i\right)$, where $B_{e}$ is another pec model and $i$ is an immersion from $A_{e}$ into $B_{e}$.

Proof Since every pec model is an amalgamation basis, the corollary follows from Theorem 1.

The following lemma and its corollary, fundamental for Section 4, are also of independent interest.

Lemma $5 \quad$ Let $A$ be an $L$-structure, let $B$ be a model of $T k(A)$, and let $C$ be an $L$-structure in which $A$ is immersed. Then there exist a model $D$ of $T k(C)$ and two immersions $\varphi$ and $\psi$ such that the following diagram commutes:

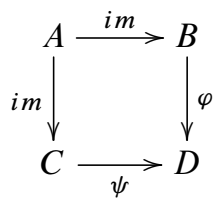

Proof We name the elements of $A$ in $B$ and $C$ by the same symbols, and we note $L^{\star}$ as the enlarged language. The proof of the theorem consists in showing that the following set of $h$-inductive sentences is consistent:

$$
\Gamma=T k(C) \cup T u(B) \cup \operatorname{Diag}^{+}(B) .
$$

Let $F=\{\chi, f(\bar{\beta}, \bar{b}), \neg \exists \bar{y} g(\bar{y}, \bar{b})\}$ be a finite subset of $\Gamma$ where $\chi \in T k(C)$, $f(\bar{\beta}, \bar{b}) \in \operatorname{Diag}^{+}(B)$ and $\neg \exists \bar{y} g(\bar{y}, \bar{b}) \in T u(B) . \quad$ As $B \models \neg \exists \bar{y} g(\bar{y}, \bar{b})$ and $B \models \exists \bar{x} f(\bar{x}, \bar{b})$, we conclude that the $h$-inductive sentence

$$
\forall \bar{z}[\exists \bar{x} f(\bar{x}, \bar{z}) \rightarrow \exists \bar{y} g(\bar{y}, \bar{z})]
$$

does not belong to $T k(B)$, and thus nor to $T k(A)$. This implies that one can find $\bar{a} \in A$ such that $A \models \neg \exists \bar{y} g(\bar{y}, \bar{a})$ and $A \models \exists \bar{x} f(\bar{x}, \bar{a})$. It follows that we can interpret the sentences in $A$ and thus in $C$, and hence conclude that $\Gamma$ is consistent. 
Corollary $3 \quad$ Let $A$ be an L-structure, and let $B$ be a model of $T k(A)$. Then every model of $T k(A)$ is immersed in a model of $T k(B)$, and every model of $T k(B)$ is immersed in a model of $T k(A)$.

\section{Hausdorff-Type Spaces and Elementary Extensions}

This section is devoted to the analysis of topological properties of spaces of positive types of an $h$-inductive theory. The main theorem-answering a question of Poizatis concerned with the Hausdorff property of type spaces. Its proof depends heavily on amalgamation techniques developed in earlier sections.

Definition $8([9$, Section 3]) An $h$-inductive theory $T$ (resp., a structure $M$ ) is said to be Hausdorff if and only if for every natural number $n$, the space $S_{n}(T)$ (resp., $S_{n}(M)$ ) is Hausdorff.

Such a definition would be useless if negation were in the language. But the exclusion of negation, which makes the topology of $S_{n}$ closer to the Zariski topology in algebraic geometry, yields rapidly examples of $h$-inductive theories whose type spaces are not Hausdorff (see the example after Lemma 6).

A natural question is the connection between the Hausdorff property of an $h$ inductive theory and those of its individual models. This necessitates the analysis of the preservation of the Hausdorff property when one goes to elementary extensions or restrictions. An affirmative answer concerning the passage to elementary extensions was proved by Poizat in [9]. The main result of this section gives an affirmative answer for passage to elementary substructures.

We start with a technical notion introduced in [4, Section 8.5].

Definition 9 Let $T$ be an $h$-inductive theory, and let $\varphi$ be a positive formula. The resultant of $\varphi$, denoted by $\operatorname{Res}_{T}(\varphi)$, is the set of positive formulas $\psi$ such that $T \vdash \neg \exists x(\varphi(x) \wedge \psi(x))$.

Lemma 6 Let $T$ be an h-inductive theory, and let $S_{n}(T)$ be its space of $n$-types. Then $S_{n}(T)$ is Hausdorff if and only if for every pair of distinct types $p, q \in S_{n}(T)$, there exist two positive formulas $f$ and $g$ such that $p \vdash f, q \vdash g$, and every formula in $\operatorname{Res}_{T}(f)$ is contradictory to every formula in $\operatorname{Res}_{T}(g)$.

Proof Let $O_{f}$ and $O_{g}$ be two basic open sets in $S_{n}(T)$; in other words, $O_{f}=\left\{r \in S_{n}(T): r \nvdash f\right\}$, and similarly $O_{g}=\left\{r \in S_{n}(T): r \nvdash g\right\}$. Equivalently, $O_{f}=\bigcup_{h \in \operatorname{Res}_{T}(f)} F_{h}$, where $F_{h}$ is nothing but the closed set defined by $h$, and similarly for $O_{g}$. The topology on $S_{n}(T)$ is Hausdorff if and only if there exist $f, g$ such that $p \vdash f, q \vdash g$ and $O_{f} \cap O_{g}=\emptyset$. This is equivalent to the conclusion of the lemma.

Before going any further, we will use this lemma to illustrate an example of a nonHausdorff theory. A slightly different version of this example was given at the end of [3]. Let $L=\left\{R_{i}: i<\omega\right\}$ be relational language. The $h$-inductive theory $T$ assures that for every $i<\omega, \operatorname{Res}_{T}\left(R_{i}\right)$ contains all but finitely many of the $R_{j}, j \neq i$. Then, by Lemma 6, $T$ is not Hausdorff.

In [3], the following characterization of the Hausdorff property of type spaces was shown. 
Fact 10 ([3, Théorème 20]) The spaces of type of an $h$-inductive theory are Hausdorff if and only if one can amalgamate the homomorphisms between models of the Kaiser envelope $T k$; that is, for any three models $M_{1}, M_{2}$, and $M_{3}$ of $T k$, such that there is a homomorphism $f$ from $M_{1}$ to $M_{2}$ and a homomorphism $g$ from $M_{1}$ to $M_{3}$, there exist $M_{4}$ a model of $T k$ and $s, h$ homomorphisms such that the following diagram commutes:

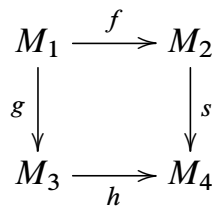

The following corollaries offer examples of Hausdorff $h$-inductive theories.

Corollary 4 Every model-complete h-inductive theory is Hausdorff.

Proof As $T$ is model-complete, by definition its class of pec models is elementary and axiomatized by $T k$. Consequently, every model of $T k$ is pec. The conclusion follows from Corollary 1 and Fact 10.

Corollary 5 An h-inductive theory that has the amalgamation property is Hausdorff.

Corollary 6 Let $L$ be a relational language, and let $T$ be a finitely axiomatizable h-universal theory. Then $T$ is Hausdorff.

Proof By Fact 7, $T k$ is model-complete. The conclusion follows from Corollary 4.

We use this corollary to verify that an example in Kungozhin [6, Example 4] is a Hausdorff theory. Let $L$ be a language that contains a single relational predicate $R$, and let $T$ be the $h$-universal theory $\{\neg \exists x y R(x, y) \wedge R(y, x)\}$. By Corollary 6, $T$ is Hausdorff.

We will now attack the question of preservation of Hausdorffness. In [9], Poizat makes the following remark.

Fact 11 ([9, Section 3]) An elementary extension of a Hausdorff structure is also Hausdorff.

The reverse implication was left as an open problem in [9]. Theorem 2 below answers affirmatively this question. Amalgamation in classes of models of Kaiser envelopes will be a major tool in the proof (see Lemma 5 and Corollary 3 ).

Theorem 2 An elementary substructure of a Hausdorff structure is Hausdorff.

Proof Let $M$ be an elementary substructure of $N$. We assume that $N$ is a Hausdorff structure. The main point of the proof will be to replace models of $T k(M)$ with models of $T k(N)$ in order to be able to use the amalgamation property of the latter and Fact 10.

Let $M_{1}, M_{2}, M_{3}$ be three models of $T k(M)$, and let $\varphi_{2}$ (resp., $\varphi_{3}$ ) be a homomorphism from $M_{1}$ to $M_{2}$ (resp., from $M_{1}$ to $M_{3}$ ). By Corollary 3, there exists $N_{1}$, 
a model of $T k(N)$ such that $M_{1}$ is immersed in $N_{1}$ and that the following diagram commutes:

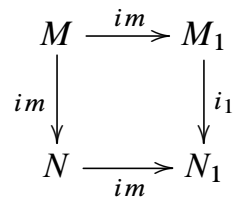

As $M_{1}$ is immersed in $N_{1}$, an application of the asymmetric amalgamation yields the following commutative diagram:

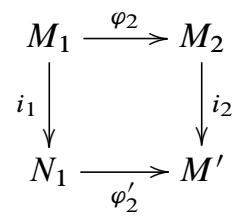

with $M^{\prime}$ a model of $T k\left(M_{2}\right)$, and thus a model of $T k(M)$. The following commutative diagram illustrates this:

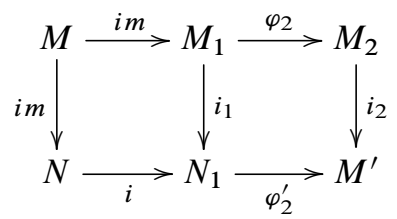

On this diagram, one remarks that the mapping $\varphi_{2}^{\prime} \circ i$ defined from $N$ to $M^{\prime}$ is an immersion because $N$ is a pec model of $T k(M)$ and $M^{\prime} \models T k(M)$, which implies that $M^{\prime}$ is a model of $T u(N)$. This allows us to find a continuation $N_{2}$ of $M^{\prime}$ that is a pec model of $T u(N)$ (Fact 3). Since it is pec, it is also a model of $T k(N)$. We thus obtain the following commutative diagram:

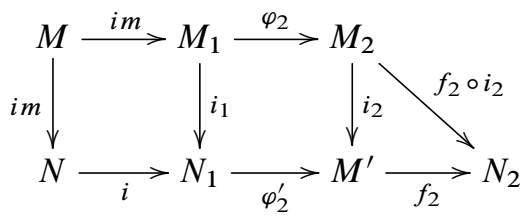

We repeat the same construction for $M_{3}$ and obtain the following commutative diagram:

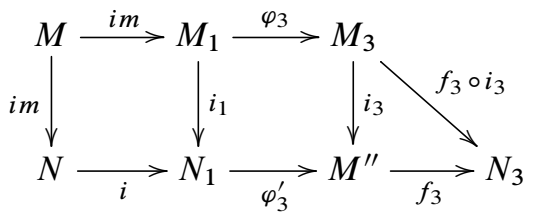


where $M^{\prime \prime}$ is a model of $T k(M), N_{3}$ is a model of $T k(N)$, and $f_{3}$ is a homomorphism. We then have the following diagram:

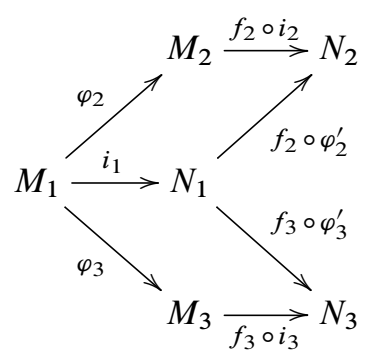

The amalgamation in models of $T k(N)$ (Fact 10) yields the following commutative diagram:

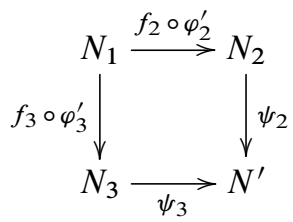

where $N^{\prime}$ is a model of $T k(N)$, and thus of $T k(M)$ as well. It follows from this that

$$
\psi_{2} \circ f_{2} \circ \varphi_{2}^{\prime} \circ i_{1}=\psi_{3} \circ f_{3} \circ \varphi_{3}^{\prime} \circ i_{1} \text {. }
$$

This implies that

$$
\psi_{2} \circ f_{2} \circ i_{2} \circ \varphi_{2}=\psi_{3} \circ f_{3} \circ i_{3} \circ \varphi_{3} .
$$

The following commutative diagram illustrates this construction:

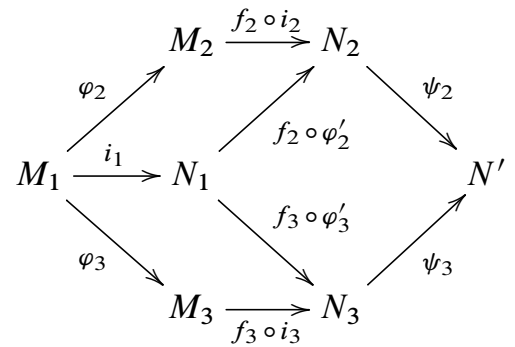

Finally, we conclude with the following commutative diagram in the class of models of $T k(M)$ :

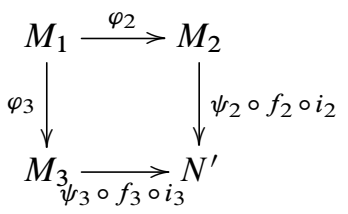

The theorem follows from Fact 10.

We end this section with an example that shows that the topology of a space of types is too weak to determine all properties of an $h$-inductive theory. The theory in question is Hausdorff, but the class of its pec models is not elementary.

Example 1 Let $L$ be the relational language $\left\{P_{i}, R_{i}: i<\omega\right\}$, and let $T$ be the $h$-universal theory 
$\left\{\neg \exists x\left(P_{i}(x) \wedge P_{j}(x)\right), \forall x\left(P_{i}(x) \vee R_{i}(x)\right), \neg \exists x\left(P_{i}(x) \wedge R_{i}(x)\right) \mid i \neq j, i, j<\omega\right\}$.

A pec model $A$ of $T$ has the following properties.

1. For every $i<\omega$ the $h$-inductive sentences $\forall x, y\left(P_{i}(x) \wedge P_{i}(y) \rightarrow x=y\right)$ belong to $T k$, because otherwise one can continue $A$ into a model of $T$ using a homomorphism that maps $x$ and $y$ to the same image. Such a homomorphism would not be an immersion and this would keep $A$ from being pec.

2. For every $i<\omega, A \models \exists x P_{i}(x)$. Indeed, if $A$ satisfies the $h$-universal sentence $\neg \exists x P_{i}(x)$, then the mapping from $A$ to $A \cup\{b\}$ where $b$ realizes $P_{i}(x)$ is not an immersion although it is an embedding. Note that $A \cup\{b\}$ is also a model of $T$. It follows that $A$ is not a pec model. Thus, for every $i<\omega$, $A \models \exists x P_{i}(x)$. In particular, $A$ is necessarily infinite.

3. In addition to the sentences in $T, A$ also satisfies the following $h$-inductive sentences:

$$
\left\{\exists x P_{i}(x), \exists x R_{i}(x), \forall x y\left(P_{i}(x) \wedge P_{i}(y)\right) \rightarrow x=y \mid i<\omega\right\} .
$$

There exist exactly two pec models of $T$, namely, $A=\left\{a_{i} \mid i<\omega\right\}$ and $B=A \cup\{x\}$, with the following properties: for every $i, j<\omega$ and $i \neq j$

$$
A, B \models P_{i}\left(a_{i}\right) \wedge R_{j}\left(a_{i}\right) \quad \text { and } \quad B \models R_{i}(x) .
$$

Now, it follows from the classical Löwenheim-Skolem theorem that the class of the pec models of $T$ is not elementary. Indeed, if it were, then for every cardinal $\lambda \geq \boldsymbol{\aleph}_{0}$, there would exist a pec model of cardinality $\lambda$.

Finally, we will show that $T$ is Hausdorff. By property (3) above of the pec models of $T$, the Kaiser envelope $T k$ associated to $T$ contains the sentences mentioned in (3). This implies that every model of $T k$ is of the form $\left\{a_{j} \mid j<\omega\right\} \cup C$, where $C$ is a possibly empty set of points each of which satisfies $R_{i}$ and none of which satisfies $P_{i}$ for every $i<\omega$.

The amalgamation in the class of models of $T k$ is done by compressing the points in $C$ as follows. Let $M_{1}, M_{2}, M_{3}$ be models of $T k$, and let $f_{2}$ (resp., $f_{3}$ ) be a homomorphism from $M_{1}$ to $M_{2}$ (resp., from $M_{1}$ to $M_{3}$ ). Since $M_{1}, M_{2}, M_{3} \models T k$, there exist $B_{1}, B_{2}, B_{3}$ such that $M_{i}=\left\{a_{j} \mid j<\omega\right\} \cup B_{i}$, and $f_{i}$ fixes pointwise the set $\left\{a_{j} \mid j<\omega\right\}$ while $f_{i}\left(B_{1}\right)=B_{i}$ for $i=2,3$. To amalgamate one uses the model $N=\left\{a_{j} \mid j<\omega\right\} \cup\{x\}$ of $T k$ and the homomorphisms $g_{i}(i=2,3)$ that fix each $a_{j}$ and map the corresponding $B_{i}$ onto $x$. The resulting diagram is commutative:

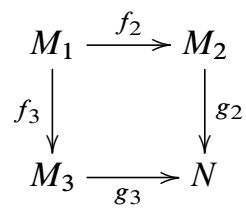

The Hausdorff property now follows from Fact 10.

The theory $T$ is an example of a Hausdorff theory whose pec models do not form an elementary class.

\section{Positive Robinson Theories and Quantifier Elimination}

In this section, we will discuss quantifier elimination in the positive context. The determination of positive types by their quantifier-free parts will play an important 
role. More generally, the "density" of the quantifier-free positive formulas within the set of positive formulas satisfied by an element in an arbitrary model of an $h$ inductive theory characterizes the general notion of elimination (Definition 13).

The characterizations of quantifier elimination vary according to the classes of models and companion theories in question. In the case where the analysis is done within the class of pec models, one deals with a positive Robinson theory, notion of which precursors are in [5] and [1] (see in particular Lemmas 8 and 9 below). In the general case, a similar analysis is carried out on all models of an $h$-inductive theory (Definition 13), and the final characterization for the $h$-universal theories is obtained using Theorem 3.

By definition, an embedding is an equivalence of quantifier-free types. Hence, in the case of a theory that assigns heavier weight to its quantifier-free formulas, it is natural that embeddings are closer to immersions than in general. This aspect of elimination is described by the notions of an $h$-maximal model (Definition 10 ) and of a weakly pec model (Definition 12).

Definition 10 ([6, Definition 4]) Let $T$ be an $h$-inductive theory. A model $A$ of $T$ is said to be h-maximal if every homomorphism from $A$ to another model of $T$ is an embedding.

\section{Lemma 7 The class of h-maximal models of an h-inductive theory is inductive.}

Proof Let $T$ be an $h$-inductive theory, let $\alpha$ be a limit ordinal, and let $\left\{M_{i} ; f_{i j} \mid\right.$ $i \geq j, i, j<\alpha\}$ be an inductive family of $h$-maximal models of $T$ of which the inductive limit will be denoted $M$.

We will show that $M$ is $h$-maximal. Since $M$ is an inductive limit of models of $T, M$ is model of $T$ (Fact 2). Let $N \models T$, and let $f$ be a homomorphism from $M$ to $N$. For every $\bar{m} \in M$, there exists $i<\alpha$ such that $\bar{m} \in M_{i}$. Let $h_{i}$ be the canonical homomorphism from $M_{i}$ into $M$, and suppose that $N \models \varphi(f(\bar{m}))$, where $\varphi$ is a free positive formula. As $M_{i}$ is $h$-maximal, the homomorphism $f \circ h_{i}$ is an embedding. But $\varphi$ is a quantifier-free formula, thus $M_{i} \models \varphi(\bar{m})$ and $M \models \varphi(\bar{m})$. Hence, $f$ is an embedding.

We will denote by $T m$ the $h$-inductive theory of the $h$-maximal models of an $h$ inductive theory $T$. Note that $T u \subset T m \subset T k$.

Corollary $7 \quad$ Let $T$ be an h-inductive theory. The class of h-maximal models of $T$ is elementary if and only if it is axiomatized by $\mathrm{Tm}$.

Proof Let $T^{\prime}$ denote the theory axiomatizing the $h$-maximal models of $T$. Then $M \models T^{\prime}$ if and only if $M$ is $h$-maximal, and so $M \models T m$. Hence, $T^{\prime} \vdash T m$. As for the reverse implication, by Fact $2, T^{\prime}$ is an $h$-inductive theory. Since $T m$ is the set of $h$-inductive sentences that are true in all $h$-maximal models of $T$, one concludes that $T m \vdash T^{\prime}$.

Let $A$ be a structure, and let $\bar{a} \in A$. We will denote by $\operatorname{tps} q(\bar{a})$ the set of positive quantifier-free formulas satisfied by $\bar{a}$ in $A$.

Definition 11 Let $T$ be an $h$-inductive theory. The theory $T$ is said to be positive Robinson if it satisfies the following condition:

for any two pec models $A$ and $B$ of $T$, if $\bar{a} \in A, \bar{b} \in B$ and $\operatorname{tps} q(\bar{a}) \subset$ $\operatorname{tps} q(\bar{b})$, then $\operatorname{tp}(\bar{a})=t p(\bar{b})$. 


\section{Remarks 1}

1. This definition is equivalent to saying that in the pec models of a positive Robinson theory, the types are entirely determined by their quantifier-free parts.

2. An $h$-inductive theory is positive Robinson if and only if it has a companion that has this property.

Example 2 The theory of fields of a given characteristic is a positive Robinson theory because its maximal $h$-inductive companions have as models the algebraically closed fields of the same characteristic.

Lemma 8 An h-inductive theory $T$ is positive Robinson if and only if it satisfies the following condition:

for every pec model $A$ of $T$, for every quantifier-free positive formula $\varphi(\bar{x})$, and $\bar{a} \in A, A \models \neg \varphi(\bar{a})$ if and only if there exists a quantifier-free positive formula $\psi(\bar{x})$ such that $A \models \psi(\bar{a})$ and $T \vdash \neg \exists \bar{x} \psi(\bar{x}) \wedge \varphi(\bar{x})$.

Proof $\quad$ Let $A$ be a pec model of $T$, and let $\bar{a} \in A$. Let us suppose that $A \models \neg \varphi(\bar{a})$. This implies that $\varphi(\bar{x})$ does not belong to the type of $\bar{a}$. We first show that $T \cup \operatorname{tps} q(\bar{a}) \cup\{\varphi(\bar{a})\}$ is inconsistent. If not, then there exist a pec model $B$ and $\bar{b} \in B$ such that $B \models \varphi(\bar{b})$ and $\operatorname{tps} q(\bar{a}) \subset \operatorname{tp}(b)$. Since $T$ is positive Robinson, $\operatorname{tp}(\bar{a})=\operatorname{tp}(\bar{b})$, which is a contradiction. It follows that there exists a finite subset $\psi(\bar{x})$ of $\operatorname{tps} q(\bar{a})$ such that $T \vdash \neg \exists \bar{x} \psi(\bar{x}) \wedge \varphi(\bar{x})$.

For the reverse direction, we assume that for every positive formula $\varphi, \operatorname{Res}_{\varphi}$ is equivalent modulo $T$ to a set of quantifier-free positive formulas. Let $A$ and $B$ be two pec models of $T, \bar{a} \in A$ and $\bar{b} \in B$ such that $\operatorname{tps} q(\bar{a}) \subset \operatorname{tps} q(\bar{b})$. Let $\varphi$ be a positive formula such that $A \models \neg \varphi(\bar{a})$; then by hypothesis, there exists a positive quantifier-free formula $\psi(\bar{x})$ such that $A \models \psi(\bar{a})$ and $T \vdash \neg \exists \bar{x} \psi(\bar{x}) \wedge \varphi(\bar{x})$. Since $\operatorname{tpsq}(\bar{a}) \subset \operatorname{tpsq}(\bar{b}), B \models \psi(\bar{b})$. This implies that $B \models \neg \varphi(\bar{b})$. It follows that $t p(\bar{b}) \subset t p(\bar{a})$. By the maximality of positive types, we deduce that $t p(\bar{a})=t p(\bar{b})$.

Corollary $8 \quad$ Let $T$ be a positive Robinson theory, and let $A$ be a model of $T$. Then $A$ is h-maximal if and only if it satisfies the following condition:

for every quantifier-free positive formula $\varphi(\bar{x})$ and $\bar{a} \in A, A \models \neg \varphi(\bar{a})$ if and only if there exists a quantifier-free positive formula $\psi(\bar{x})$ such that $A \models \psi(\bar{a})$ and $T \vdash \neg \exists \bar{x} \psi(\bar{x}) \wedge \varphi(\bar{x})$.

Proof Let $A$ be an $h$-maximal and $B$ a pec model of $T$ such that $A$ embeds in $B$. We assume that $A \models \neg \varphi(\bar{a})$. Thus, $B \models \neg \varphi(\bar{a})$. Since $B$ is a pec model, by Lemma 8 there exists $\psi(\bar{x})$ a quantifier-free positive formula such that $T \vdash \neg \exists \bar{x} \psi(\bar{x}) \wedge \varphi(\bar{x})$ and $B \models \psi(\bar{a})$. This implies that $A \models \psi(\bar{a})$. In the reverse direction, every model of $T$ that satisfies the condition above is $h$-maximal.

Lemma 9 Let $T$ be a positive Robinson theory. Then the following conditions are satisfied.

1. Every model of $T$ that embeds in a pec model of $T$ is h-maximal.

2. The h-maximal models of $T$ have the amalgamation property.

Moreover, if $T$ is h-universal, then these two conditions are sufficient to conclude that $T$ is a positive Robinson theory. 
It is worth noting that the second condition of Lemma 9 shows that the $h$-maximal models of a positive Robinson theory are amalgamation bases.

Proof Let $A, B, C$ be three models of $T$ such that there is an embedding $i$ from $A$ into $B$ and a homomorphism $f$ from $A$ into $C$, and $B$ is a pec model. Let $C e$ be a pec model of $T$ such that there is a homomorphism $j$ from $C$ to $C e$ :

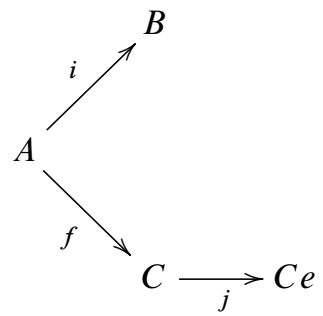

For any $\bar{a} \in A$,

$$
\operatorname{tpsq}(\bar{a})=\operatorname{tpsq}(i(\bar{a})) \text { and } \operatorname{tps} q(\bar{a}) \subset \operatorname{tps} q(f(\bar{a})) \subset \operatorname{tps} q(j \circ f(\bar{a})) .
$$

Since $T$ is a positive Robinson theory, $B$ and $C e$ are pec models of $T$, and $\operatorname{tpsq}(i(\bar{a})) \subset \operatorname{tpsq}(j \circ f(\bar{a}))$, we conclude that $\operatorname{tpsq}(i(\bar{a}))=\operatorname{tpsq}(j \circ f(\bar{a}))$. It follows that $\operatorname{tps} q(\bar{a})=\operatorname{tps} q(f(\bar{a}))$, and thus $f$ is an embedding.

We will now show the amalgamation property for $h$-maximal models. Let $A, B$, and $C$ be $h$-maximal models of $T$ with $i$ and $j$ embeddings from $A$ into $B$ and $C$, respectively. Let $B e$ and $C e$ be pec models of $T$ that are continuations of $B$ and $C$, respectively. We then have the following diagram:

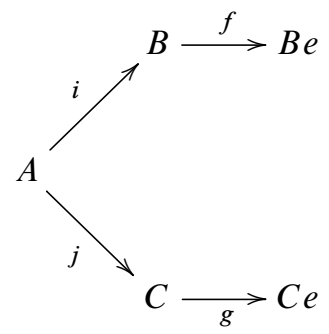

For every $\bar{a} \in A, t p s q(\bar{a})=\operatorname{tps} q(f \circ i(\bar{a}))=\operatorname{tps} q(g \circ j(\bar{a}))$. Thus, $\bar{a}$ has the same type $p$ in $B e$ and $\mathrm{Ce}$.

In order to complete the amalgamation argument, we will show using positive compactness that $T \cup D^{+}(B e) \cup D^{+}(C e)$ is consistent. Let $\varphi(\bar{a}, \bar{b}) \in D^{+}(B e)$ and $\psi(\bar{a}, \bar{c}) \in D^{+}(C e)$, where $\bar{a}$ is the tuple of parameters that belong to $A$. Then, $\exists y \varphi(x, y)$ and $\exists z \psi(x, z)$ belong to $p$. As a result, there exists $\bar{c}^{\prime} \in C e$ such that $\varphi\left(\bar{a}, \bar{c}^{\prime}\right) \wedge \psi(\bar{a}, \bar{c}) \in D^{+}(C e)$, and the consistency follows. It follows that $T \cup D^{+}(B e) \cup D^{+}(C e)$ has a model $D$ that one can continue to an $h$-maximal model of $T$ (a pec model of $T$ for example). The amalgamation property for the $h$-maximal models follows.

Now, we assume that $T$ is $h$-universal and that the two conditions in the statement hold. We will prove that $T$ is positive Robinson. Let $A$ be a pec model of $T, \bar{a}, \bar{b} \in A$ such that $\operatorname{tpsq}(\bar{a}) \subset \operatorname{tpsq}(\bar{b})$. Let $\langle\bar{a}\rangle$ be the substructure of $A$ generated by $\bar{a}$. As $T$ is $h$-universal, $\langle\bar{a}\rangle \models T$. Since the inclusion $\langle\bar{a}\rangle$ in $A$ is an embedding, $\langle\bar{a}\rangle$ is an $h$-maximal model of $T$ by condition 1 . Hence, the homomorphism $f$ from $\langle\bar{a}\rangle$ into 
$A$ that maps $\bar{a}$ to $\bar{b}$ is an embedding. The amalgamation property of the $h$-maximal models (condition 2) shows that there exists $B$ that may be chosen to be a pec model such that the following diagram commutes:

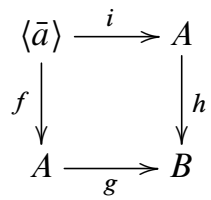

Thus $h(\bar{a})=g(\bar{b})$. Note that, since $A$ is a pec model, $g, h$ are immersions. Hence, $\bar{a}$ and $\bar{b}$ have the same type.

Remark 2 If every model of $T$ that embeds in a pec model is an $h$-maximal, then every model of $T$ that embeds in an $h$-maximal is an $h$-maximal.

Corollary 9 If $T$ is a positive Robinson theory of which the class of h-maximal models is elementary, then $T$ is Hausdorff.

Proof As $T$ is positive Robinson, its $h$-maximal models have the amalgamation property; that is, they satisfy condition 2 of Lemma 9 . Since the class of $h$-maximal models is elementary, it is axiomatized by the $h$-inductive theory $T m$ (Corollary 7). Let $M_{1}, M_{2}, M_{3}$ be models of $T k$ with $f$ and $g$ homomorphisms from $M_{1}$ to $M_{2}$ and $M_{3}$, respectively. Since $T m \subset T k$, these three models are $h$-maximal. As a result, there exist a model $N$ of $T m$ and a pec continuation $M$ of $N$ such that the following diagram commutes:

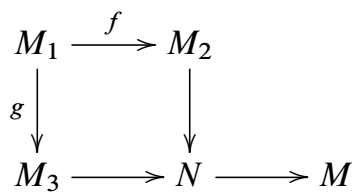

Since $M \models T k$, the Hausdorff property of $T$ follows from Fact 10 .

In the rest of this section, we will extend the preceding discussion to all models of an $h$-inductive theory. The notion of a weakly pec model and the property EQ will be crucial.

Definition 12 Let $T$ be an $h$-inductive theory. A model $A$ of $T$ is said to be weakly pec if every embedding from $A$ into a model of $T$ is an immersion.

We first refine a notation already introduced. For an $h$-inductive theory $T$, a model $M$ of $T$, and $\bar{a} \in M$, we will denote by $f_{M}(\bar{a})$ the set of quantifier-free positive formulas satisfied by $\bar{a}$ in $M$.

Definition 13 An $h$-inductive theory $T$ is said to have the property $E Q$ if it satisfies the following hypothesis:

for every pair of models $A$ and $B$ of $T, \bar{a} \in A$ and $\bar{b} \in B, f_{A}(\bar{a})=f_{B}(\bar{b})$ if and only if $F_{A}(\bar{a})=F_{B}(\bar{b})$.

The property EQ will allow us to characterize the elimination of quantifiers in $h$ universal theories. We start with a general lemma.

Lemma 10 If an h-inductive theory $T$ has the property $E Q$, then every embedding between models of $T$ is an immersion. In particular, every model of $T$ is weakly pec. 
Proof We assume that $T$ has the property EQ. Let $A$ and $B$ be two models of $T$, let $i$ be an embedding from $A$ into $B$, and let $\bar{a} \in A$. Then $\bar{a}$ and $i(\bar{a})$ satisfy the same quantifier-free positive formulas. They satisfy the same positive formulas since $T$ has the property EQ. It follows that $i$ is an immersion, and one concludes that every model of $T$ is weakly pec.

Corollary 10 If $T$ is a theory having the property $E Q$, then every h-maximal model of $T$ is pec.

Corollary 11 If $T$ is an h-universal theory having the property $E Q$, then $T$ is a positive Robinson theory.

Proof Since $T$ has the property EQ, Lemma 10 shows that every embedding between models of $T$ is an immersion. Subsequently, every model $A$ of $T$ that embeds in a pec model of $T$ is a pec model; it is in particular $h$-maximal.

By Corollary 10, every $h$-maximal model of $T$ is pec. The amalgamation property for $h$-maximal models follows from Corollary 1.

The conclusion of the corollary follows from Lemma 9.

Theorem 3 Let $T$ be an h-universal theory. Then the following conditions are equivalent:

1. T has the property $E Q$;

2. every model of $T$ is weakly pec;

3. every positive formula is equivalent modulo $T$ to a quantifier-free positive formula.

Proof $\quad(1 \Rightarrow 2)$ This is Lemma 10 .

$(2 \Rightarrow 1)$ Let $\bar{a} \in A, \bar{b} \in B$ such that $f_{A}(\bar{a})=f_{B}(\bar{b})$, and $\langle\bar{a}\rangle$ is the substructure of $A$ generated by $\bar{a}$. Since $T$ is $h$-universal, $\langle\bar{a}\rangle$ is a model of $T$. It embeds in $A$ through the inclusion mapping that we will denote by $i$ and in $B$ through the embedding that maps $\bar{a}$ onto $\bar{b}$ and that we will denote by $j$. By hypothesis 2, the embeddings $i$ and $j$ are immersions. By asymmetric amalgamation (Lemma 4), there exist a model $D$ of $T$ together with an immersion $f$ and a homomorphism $g$ that make the following diagram commute:

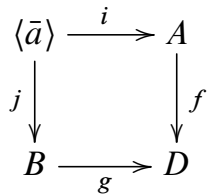

If $B \models \exists \bar{y} \varphi(\bar{b}, \bar{y})$, where $\bar{b}=j(\bar{a})$, then $B \models \exists \bar{y} \varphi(j(\bar{a}), \bar{y})$, and $D \models \exists \bar{y} \varphi(g \circ$ $j(\bar{a}), \bar{y})$. Since $f$ is an immersion, $A \models \exists \bar{y} \varphi(a, \bar{y})$, thus $F_{B}(\bar{b}) \subset F_{A}(\bar{a})$.

In order to show that $F_{A}(\bar{a}) \subset F_{B}(\bar{b})$, one redoes the same argument on the commutative diagram

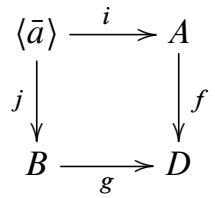

this time with $g$ as an immersion. 
$(3 \Rightarrow 2)$ We assume 3 . Then every embedding is an immersion; thus every model of $T$ is weakly pec.

The idea of the proof of $(1 \Rightarrow 3)$ is from [4, Lemma 8.4.8].

$(1 \Rightarrow 3)$ Let $\varphi$ be a positive formula, and let $\Delta$ be the set of quantifier-free positive formulas $\psi$ such that $T \vdash \varphi \rightarrow \psi$. We will show that $T \cup \Delta(x) \vdash \varphi(x)$ in the language $L \cup\{x\}$. Let $B$ be a model of $T \cup \Delta(x)$. Let $\Gamma$ be the set of quantifier-free positive formulas $\chi$ such that $B \models \neg \chi(x)$, and let $T^{\prime}$ be the theory $T \cup\{\varphi(x)\} \cup\{\neg \chi(x) \mid \chi \in \Gamma\}$.

Suppose towards a contradiction that $T^{\prime}$ is not consistent. Then there exists a quantifier-free positive formula $\chi$ such that $T \vdash \varphi(x) \rightarrow \chi(x)$. By the definition of $\Delta$, one concludes that $\chi \in \Delta$. Since $B \models \neg \chi(x)$, we reach a contradiction with the fact that $B$ is a model of $\Delta$. Hence, $T^{\prime}$ is consistent.

Let $A$ be a model of $T^{\prime}$, and let $C$ be the substructure of $A$ generated by the constants in the language $L \cup\{x\}$. So $C$ embeds in $A$, and as $T$ is $h$-universal, $C \models T$. By Lemma 10, this embedding is an immersion.

On the other hand, the mapping $j$ from $C$ to $B$ that maps every constant of $L$ onto itself and $x$ onto $x$ is a homomorphism. Indeed, suppose that $C \models \alpha(x, a)$ and $B \models \neg \alpha(x, a)$, with $x, a$ constants of the language $L \cup\{x\}$, and $\alpha$ a quantifier-free positive formula. The fact that $B \models \neg \alpha(x, a)$ implies that $\alpha(x, a) \in \Gamma$. As a result, $C \models \neg \alpha(x, a)$, which is a contradiction. Hence $j$ is a homomorphism. We obtain the following diagram:

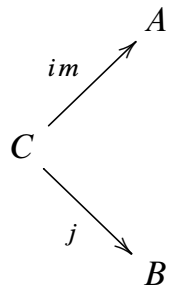

where $i m$ is an immersion and $j$ is a homomorphism. Since $A \vdash \varphi(x), C \vdash \varphi(x)$, and thus $B \vdash \varphi(x)$. Hence, $T \cup \Delta(x) \vdash \varphi(x)$.

The conclusion of the preceding paragraph and positive compactness imply that there exists $\psi \in \Delta$ such that $T \vdash \psi \rightarrow \varphi$. By the definition of $\Delta$, we conclude that $T \vdash \varphi \leftrightarrow \psi$.

\section{Complete Theories}

In this final section, we will propose a general notion of completeness for arbitrary $h$ inductive theories. In [3] Ben Yaacov and Poizat introduced the notion of a complete theory as the $h$-universal theory of a structure and showed that in the case of an $h$ universal theory this notion is equivalent to the joint continuation property defined below. In this section, we will pursue their approach and analyze completions of an arbitrary $h$-inductive theory.

Definition 14 An $h$-inductive theory $T$ is said to be complete if it has the joint continuation property:

for any two models of $T$, there exists a third model $C$ of $T$ that is a continuation of both $A$ and $B$. 
Fact 12 ([3, Lemme 7]) An $h$-inductive theory is complete if and only if it has a companion that is complete.

Lemma 11 An h-inductive theory that has a unique pec model is complete.

Proof Every model of $T$ has a pec continuation. Since there is only one such, the joint continuation condition is satisfied.

On the other hand, the following is an example of an incomplete $h$-inductive theory in our sense.

Example 3 Let $L$ be the relational language $\{Q, R\}$, and let $T$ be the $h$-universal theory $\{\neg \exists x, y Q(x) \wedge R(y)\}$. The theory $T$ is not complete since if $A$ and $B$ are models of $T$ such that $A \models R(a)$ and $B \models Q(b)$, then there does not exist a model of $T$ that is a continuation of $A$ and $B$.

We will introduce a method to obtain and characterize minimal completions (Definition 15) of an arbitrary $h$-inductive theory. The fundamental ingredient is an equivalence relation on the pec models of the theory in question. In this vein, let $T$ be an $h$-inductive theory, and let $A e$ and $B e$ be pec models of $T$. Let $\Re$ be the binary relation defined on the class of pec models of $T$ by: $A e \Re B e$ if and only if there exists a model $C$ of $T$ that is a common continuation of $A e$ and $B e$. Note that $A e \Re B e$ is also equivalent to saying that there exists a pec model $C e$ that is a common continuation of $A e$ and $B e$.

\section{Lemma 12 The relation $\Re$ is an equivalence relation.}

Proof It is easy to see that $\Re$ is reflexive and symmetric. It remains therefore to check the transitivity property. Let $A e, B e, C e$ be pec models of $T$ such that $A e \Re B e$ and $B e \Re C e$. Then we have the following diagram:

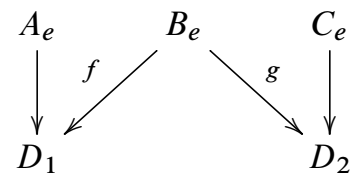

where $D_{1}$ and $D_{2}$ are models of $T$. Since $B e$ is pec, $f$ and $g$ are immersions. By asymmetric amalgamation,

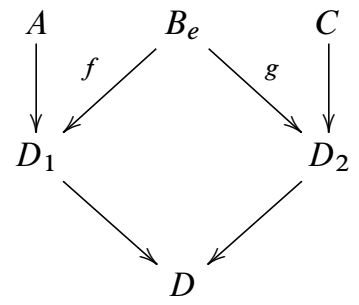

with $D \models T$. Thus, $A e \Re C e$, and it follows that $\Re$ is an equivalence relation.

Let $\mathcal{E}$ denote an equivalence class of $\Re$. We define a subclass of models of $T$ denoted $\Gamma_{\mathscr{E}}$ :

$\Gamma_{\mathcal{E}}=\{A \models T: A$ has a continuation that is a member of $\mathcal{E}\}$. 
Lemma 13 The members of $\&$ have the same h-universal theory.

Proof Let $A e$ and $B e$ be in $\mathcal{E}$. By definition, there exists a model $C$ of $T$ in which $A e$ and $B e$ are immersed (they are pec models of $T$ ). Hence, if $B e \models \neg \exists \bar{x} \varphi(\bar{x})$, then $C \models \neg \exists \bar{x} \varphi(\bar{x})$, and similarly for $A e$. This implies that $A e$ and $B e$ have the same $h$-universal theory.

We will denote by $T u(\mathcal{E})$ the $h$-universal theory found in the preceding lemma.

Lemma 14 The class $\Gamma_{\mathcal{E}}$ is axiomatized by the theory $T_{\mathcal{E}}=T \cup T u(\mathcal{E})$.

Proof We will first show that all models of $T_{\mathcal{E}}$ belong to $\Gamma_{\mathcal{E}}$. Let $A$ be a model of $T_{\mathcal{E}}$ and $B e$ in $\mathcal{E}$. Then the family $T_{\mathcal{E}}^{\prime}=T_{\mathcal{E}} \cup \operatorname{Diag}^{+}(A) \cup \operatorname{Diag}^{+}(B e)$ is consistent. For every formula $\exists \bar{x} \varphi(\bar{x}, \bar{a}) \in \operatorname{Diag}^{+}(A), \neg \exists x y \varphi(x, y)$ does not belong to $T u(\mathcal{E})$ because $A \models T_{\mathcal{E}}$. Moreover, since $B e$ belongs to $\mathcal{E}, T u(\mathcal{E})$ is the $h$-universal theory of $B e$ by Lemma 13. One can thus find $\bar{b} \in B e$ such that $B e \models \exists x \varphi(x, \bar{b})$. Hence, $T_{\mathcal{E}}^{\prime}$ is consistent, and one deduces from this that $A$ and $B e$ have a joint continuation $C$ that we may continue in a pec model $C e$ of $T$. It then follows that $B e \Re C e$, and thus $C e \in \mathcal{E}$. Hence, $A \in \Gamma_{\mathcal{E}}$.

As for the reverse implication, if $A \in \Gamma_{\mathcal{E}}$, then there exists $A e \in \mathcal{E}$, a continuation of $A$, which forces that $A \models T_{\mathcal{E}}$. One concludes from this that $\Gamma_{\mathcal{E}}$ is an elementary class axiomatized by $T_{\mathcal{E}}$.

Corollary 12 The theory $T_{\mathcal{E}}$ of Lemma 14 is complete.

Proof Let $A$ and $B$ be two models of $T_{\mathcal{E}}$. By Lemma 14, $A, B \in \Gamma_{\mathcal{E}}$. By the definition of $\Gamma_{\mathcal{E}}$, there exist two pec models $A e$ and $B e$ of $T$ in $\varepsilon$ that are continuations of $A$ and $B$, respectively. By the definition of $\mathcal{E}, A e$ and $B e$ have a common continuation in $\mathcal{E}$. The conclusion follows.

Corollary 13 The class $\Gamma_{\mathcal{E}}$ is inductive.

Proof The conclusion follows from Fact 2 and Lemma 14.

Definition 15 Let $T$ be an $h$-inductive theory. A theory $T^{\prime}$ is said to be a minimal completion of $T$ if $T^{\prime}$ is a complete theory that contains $T$ and has as model a pec model of $T$, and it is minimal with respect to these properties.

Corollary 14 The theory $T_{\mathbb{E}}$ is a minimal completion of $T$. Moreover, there exists a bijective correspondence between the equivalence classes of $\Re$ and the minimal completions of $T$.

Proof We start by verifying the first assertion. By Corollary $12, T_{\mathcal{E}}$ is complete. Its pec models include the members of $\mathcal{E}$. Finally, $T_{\mathcal{E}}$ is minimal since it is exactly $T \cup T u(\varepsilon)$.

Now, we prove the second assertion. We first define the correspondence. We associate to each class $\mathscr{E}$ of $\Re$ the theory $T_{\mathcal{E}}$. Clearly this is well defined and surjective by the very definition of a minimal completion of an $h$-inductive theory (Definition 15).

We will next verify the injective property. The main step in the proof is to prove that the pec members of $\Gamma_{\mathcal{E}}$ are exactly the members of $\mathcal{E}$. By definition, every element of $\mathscr{E}$ is a pec model of $T$, thus it is a pec member of $\Gamma_{\mathcal{E}}$. As for the other inclusion, let $A$ be a pec member of $\Gamma_{\mathcal{E}}$ that has as continuation a model $B$ of $T$, and let $f$ be the witnessing homomorphism from $A$ to $B$. By definition of $\Gamma_{\mathcal{E}}, A$ has 
a continuation $A e$ that belongs to $\mathcal{E}$. As $\mathcal{E}$ is contained in $\Gamma_{\mathcal{E}}$, the homomorphism from $A$ into $A e$, say, $g$, is in fact an immersion. By asymmetric amalgamation, there is a model $C$ of $T$ such that the following diagram commutes:

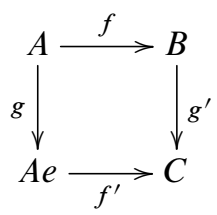

Since $g$ and $f^{\prime}$ are immersions, it follows that $f$ is an immersion. Thus $A \in \mathcal{E}$. This finishes the proof of the main step, from which the injectivity follows rapidly. Indeed, if $T_{\mathcal{E}_{1}}=T_{\mathcal{E}_{2}}$, then $\Gamma_{\mathcal{E}_{1}}=\Gamma_{\mathcal{E}_{2}}$, and the main step shows that $\mathcal{E}_{1}=\mathcal{E}_{2}$.

\section{References}

[1] Ben Yaacov, I., "Positive model theory and compact abstract theories," Journal of Mathematical Logic, vol. 3 (2003), pp. 85-118. MR 1978944. DOI 10.1142/S0219061303000212. 205, 221

[2] Ben Yaacov, I., "Simplicity in compact abstract theories," Journal of Mathematical Logic, vol. 3 (2003), pp. 163-91. MR 2030083. DOI 10.1142/S0219061303000297. 205

[3] Ben Yaacov, I., and B. Poizat, "Fondements de la logique positive," Journal of Symbolic Logic, vol. 72 (2007), pp. 1141-62. MR 2371196. DOI 10.2178/js1/1203350777. 205, 206, 207, 208, 210, 211, 214, 215, 216, 217, 226, 227

[4] Hodges, W., Model Theory, vol. 42 of Encyclopedia of Mathematics and its Applications, Cambridge University Press, Cambridge, 1993. MR 1221741. DOI 10.1017/CBO9780511551574. 205, 211,216, 226

[5] Hrushovski, E., "Simplicity and the Lascar group," preprint, 1997. 205, 221

[6] Kungozhin, A., "Existentially closed and maximal models in positive logic" (in Russian), Algebra i Logika, vol. 51 (2012), pp. 748-65; English translation in Algebra and Logic, vol. 51, no. 6 (2013), pp. 496-506. MR 3088140. 208, 217, 221

[7] Pillay, A., "Forking in the category of existentially closed structures," pp. 23-42 in Connections between Model Theory and Algebraic and Analytic Geometry, vol. 6 of Quaderni di Matematica, Dept. Math., Seconda Univ. Napoli, Caserta, 2000. MR 1930681. 205, 211

[8] Poizat, B., “Univers positifs," Journal of Symbolic Logic, vol. 71 (2006), pp. 969-76. MR 2251549. DOI 10.2178/jsl/1154698585. 205, 209

[9] Poizat, B., "Quelques effets pervers de la positivité," Annals of Pure and Applied Logic, vol. 161 (2010), pp. 812-16. MR 2601034. DOI 10.1016/j.apal.2009.06.012. 205, 208, 209, 216, 217

[10] Shelah, S., "The lazy model-theoretician's guide to stability," pp. 241-308 in Comptes Rendus de la Semaine d'Étude en Théorie des Modèles (Louvain-la-Neuve, 1975), vol. 18 of Logique et Analyse (N.S.), 1975. MR 0539969. 205 
Université de Lyon

Université Lyon 1

CNRS UMR 5208 Institut Camille Jordan

43 blvd du 11 novembre 1918

69622 Villeurbanne cedex

France

belkasmi@math.univ-lyon1.fr 